\title{
Surface modification of cellulose using silane coupling agent
}

\author{
Manju Kumari Thakur ${ }^{1}$, Raju Kumar Gupta ${ }^{2}$ and Vijay Kumar Thakur ${ }^{3 *}$ \\ ${ }^{1}$ Division of Chemistry, Govt. Degree College Sarkaghat, Himachal Pradesh University Shimla - \\ 171005, INDIA \\ ${ }^{2}$ Department of Chemical Engineering, Indian Institute of Technology Kanpur, 208016, INDIA \\ ${ }^{3}$ School of Mechanical and Materials Engineering, Washington State University, U.S.A \\ Email: vijayisu@hotmail.com; shandilyamn@gmail.com; guptark@iitk.ac.in; Phone: 0512-259- \\ 6972; Fax: 0512 - 259-0104
}




\begin{abstract}
Recently there has been a growing interest in substituting traditional synthetic polymers with natural polymers for different applications. However, natural polymers such as cellulose suffer from few drawbacks. To become viable potential alternatives of synthetic polymers, cellulosic polymers must have comparable physico-chemical properties to that of synthetic polymers. So in the present work, cellulose polymer has been modified by a series of mercerization and silane functionalization to optimize the reaction conditions. Structural, thermal and morphological characterization of the cellulose has been done using FTIR, TGA and SEM, techniques. Surface modified cellulose polymers were further subjected to evaluation of their properties like swelling and chemical resistance behavior.
\end{abstract}

Key Words: Natural cellulose; surface modification; thermal; morphological and physicochemical properties. 


\section{Introduction}

Synthetic polymers have been found to play an important role in a number of applications(Zhou et al., 2013)(Ding et al., 2013)(Chen \& Gao, 2007b). These polymer have been used as diferent components in the energy storage systems starting form electrolyte to dielectric capacitors for energy storage applications(Chen \& Gao, 2007a)(Lin \& Lee, 2013)(Lin, Thakur, Tan, \& Lee, 2011a). However, during the last few decades, environmental friendly natural polymers have attracted greater attention all over the world due to the increasing environmental awareness as well as the depletion of petroleum based resources (Banerjee et al., 2013)(Bharti, Mishra, \& Sen, 2013)( Thakur, Thakur, \& Gupta, 2014)( Thakur, Thakur, \& Gupta, 2013a). Due to their inherent properties such as renewable nature, biodegradability, economic/ low cost, easy availability, natural polymers are emerging as viable alternatives to traditional petroleum-based materials (G. U. Rani, Mishra, Sen, \& Jha, 2012)( Thakur, Thakur, \& Gupta, 2013c)(Basta, ElSaied, El-Hadi, \& El-Dewiny, 2013). Among various types of natural polymers, cellulose based polymers are the most abundant natural materials available in earth(Mishra, Rani, \& Sen, 2012)( Thakur, Thakur, \& Gupta, 2013b). Different types of biopolymers ranging from algae to natural cellulosic fibers can be the best option to serve the current energy needs (Banerjee et al., 2013)(Jawaid \& Khalil, 2011)(Sabo, Basta, \& Winandy, 2013). The effective use of biorenewable cellulosic polymers, which have been shown to have some promising properties comparable to traditionally used synthetic polymers can successfully reduce the use of petroleum resources(P. Rani, Sen, Mishra, \& Jha, 2012)(Dhakal, Zhang, Guthrie, MacMullen, \& Bennett, 2013)(Thakur, Singha, \& Thakur, 2011). Natural cellulosic polymers such as cellulosic fibers are typically derived from different types of plants, grasses and 
various other resources(Thakur, Singha, \& Thakur, 2013a). Cellulose fibers exhibit the potential to replace traditional synthetic fibers as reinforcement in the production of cellulose-fiberreinforced green composites(Khoathane, Vorster, \& Sadiku, 2008)(Thakur, Singha, \& Thakur, 2013b)(Thakur, Singha, \& Thakur, 2012). These cellulosic composites are being envisioned as one of the growing sectors in the composites industry due to their lower density, high specific modulus/ strength, and low economic cost than synthetic fibers reinforced composites(Dhakal, Zhang, Reis, Surip, \& Bennett, 2012)(Khalil et al., 2013).

Among various cellulosic fibers Eulaliopsis binata fibers possess a strong potential to be used as reinforcement in composites(Thakur, Thakur, \& Gupta, 2013c)(Thakur, Thakur, \& Gupta, 2013e) (Thakur, Thakur, \& Gupta, 2013f). These fibers can be used to prepare lightweight composites and in many other applications. However, the hydrophilic nature of these cellulosic fibers limits their applications as it results in poor chemical as well as moisture absorption resistance (Thakur, Thakur, \& Gupta, 2013c). These properties in the long term, affect the overall properties of the composite materials. So in this preliminary work, we have performed the surface modification of cellulosic Eulaliopsis binata fibers through a series of mercerization and silane functionalization. 


\section{EXPERIMENTAL}

\subsection{Materials and methods}

Natural Cellulosic Eulaliopsis binata fibers used in this preliminary work were obtained from local resources of Himalayan region. Prior to use, these fibers were purified as per standard method(Thakur, Thakur, \& Gupta, 2013c)(Thakur, Thakur, \& Gupta, 2013e). In brief, these fibers were initially soaked in a detergent solution for specific time intervals and subsequently extensively washed with tap water. Chemicals namely, ethanol, acetone, sodium hydroxide $(\mathrm{NaOH})$, and vinyltrimethoxysilane were used as received.

The surface modification of the Eulaliopsis binata fibers was initiated by first carrying out the mercerization of these fibers(Singha et al., 2009). Mercerization is frequently referred as the pretreatment process in the modification of biopolymers(Singha \& Thakur, 2009a). In the mercerization process, cellulosic Eulaliopsis binata fibers were immersed in pre-demined (2\%) concentration of $\mathrm{NaOH}$ solution for series of time intervals at room temperature for the optimization of time. Subsequently, these fibers were thoroughly washed with distilled water for removal of any $\mathrm{NaOH}$ content, followed by neutralization with $2 \%$ acetic acid and excess washing with water(Singha \& Thakur, 2009b). After the completion of this reaction step, these fibers were dried in an oven at $70{ }^{\circ} \mathrm{C}$ to a constant weight. After the optimization of time, the effect of different concentration of $\mathrm{NaOH}$ solutions (2-14\%) at the optimized time period was also studied. The optimization of time and concentration of $\mathrm{NaOH}$ ensures that cellulosic fibers are ready for modification with silane treatment(Thakur, Singha, Kaur, Nagarajarao, \& Liping, 2010). 
Surface functionalization of the Eulaliopsis binata fibers was done using vinyltrimethoxysilane as the coupling agent. The mercerized Eulaliopsis binata fibers were dipped in an ethanol/water mixture in the ratio (60:40) with optimized concentration (5\%) of the coupling agent for $2 \mathrm{~h}$. In order to ensure efficient coating of vinyltrimethoxysilane coupling agent on the fibers, the $\mathrm{pH}$ of the solution was maintained between 3.5 and 4 , using the METREPAK Phydrion buffers(Singha et al., 2009) (Singha \& Thakur, 2009a) (Singha \& Thakur, 2009b). After the predetermined time interval, the ethanol/water mixture was drained out. The Eulaliopsis binata fibers fibres were then dried in air and then in an oven at $70{ }^{\circ} \mathrm{C}$ to a constant weight(Singha et al., 2009) (Singha \& Thakur, 2009a) (Singha \& Thakur, 2009b).

\subsection{Structural and physico-chemical characterization}

FT-IR spectra of the pristine and vinyltrimethoxysilane treated Eulaliopsis binata fibers taken using $\mathrm{KBr}$ pellets on PERKIN ELMER RXI Spectrophotometer in the range of 400-4000 $\mathrm{cm}^{-1}$. The surface morphology of the pristine/ silane treated Eulaliopsis binata fibers was studied Scanning electron microscopic (SEM), while the thermal (TGA) behaviors was were studied with the help of a thermal analyzer (Perkin Elmer) at a heating rate of $10{ }^{\circ} \mathrm{C} / \mathrm{min}$ by taking $10 \mathrm{mg}$ weight for each sample in nitrogen atmosphere(Singha et al., 2009) (Singha \& Thakur, 2009a) (Singha \& Thakur, 2009b). The effect of silane coupling agent on the swelling/ water uptake behavior of the pristine/ silane treated Eulaliopsis binata fibers was studied using the standard method in different solvents e.g. water, methanol, and isobutanol(Singha et al., 2009) (Singha \& Thakur, 2009a) (Singha \& Thakur, 2009b). The percent swelling behaviors of the pristine/ vinyltrimethoxysilane treated Eulaliopsis binata fibers was determined in the following manner(Singha et al., 2009) (Singha \& Thakur, 2009a) (Singha \& Thakur, 2009b): 


$$
\text { Percent swelling }\left(P_{S}\right)=\frac{W_{f}-W_{i}}{W_{i}} \times 100
$$

Where $\mathrm{W}_{\mathrm{f}}=$ Final weight of the Eulaliopsis binata fibers

$$
\mathrm{W}_{\mathrm{i}}=\text { Initial weight of the Eulaliopsis binata fibers }
$$

The effect of silane coupling agent on the chemical resistance behavior of the pristine/ silane treated Eulaliopsis binata fibers was studied as per the method standard method in the following manner(Singha et al., 2009) (Singha \& Thakur, 2009a) (Singha \& Thakur, 2009b):

$$
\text { Percent chemical resistance }\left(P_{c r}\right)=\frac{T_{w}-W_{a c i}}{T_{w}} \times 100
$$

Where $T_{w}=$ total weight of the Eulaliopsis binata fibers

$$
W_{a c i}=\text { weight after certain interval Eulaliopsis binata fibers }
$$

\section{Results and Discussion}

Surface modification of materials procured from different natural and synthetic resources through different treatments is one of the facile method to obtain the materials with desired properties(Dhakal, Zhang, \& Bennett, 2012)(Lin, Thakur, Tan, \& Lee, 2011b)(Pilla, Gong, O’Neill, Rowell, \& Krzysik, 2008). There is some existing literature available on the effect of surface treatment on the mechanical properties of polymer composites but limited information is available on the thorough study of effect of vinyltrimethoxysilane treatment on the 
physicochemical properties of the Eulaliopsis binata fibers(Pilla, Gong, O’Neill, Yang, \& Rowell, 2009)(Maheswari, Reddy, Muzenda, Shukla, \& Rajulu, 2013). So in this work we have studied the effect of vinyltrimethoxysilane treatment on the physico-chemical properties such as water absorption, water uptake and chemical resistance of the Eulaliopsis binata fibers. The hydroxy groups present in Eulaliopsis binata fibers backbone have been found to responsible for the water/ moisture absorption (Thakur, Thakur, \& Gupta, 2013c)(Thakur, Thakur, \& Gupta, 2013e). These functional groups also play a key role in the surface modification of Eulaliopsis binata fibers as these are consist of linear glucan chains with repeating $(1 \rightarrow 4) \beta$-glucopyranose units (Pinto, Chalivendra, Kim, \& Lewis, 2014)(Pinto, Chalivendra, Kim, \& Lewis, 2013) (Thakur, Thakur, \& Gupta, 2013c)(Thakur, Thakur, \& Gupta, 2013e). Mercerization is one of the best method to remove some of the impurities from the surface of the natural cellulosic fibers. In the present work, the activation of functional groups on Eulaliopsis binata fibers have been carried out using mercerization of these fibers with different concentration of $\mathrm{NaOH}$ for selected time intervals using the standard method reported in the literature (Singha et al., 2009) (Singha \& Thakur, 2009a) (Singha \& Thakur, 2009b). These mercerized Eulaliopsis binata fibers were subsequently subjected to surface functionalization with the vinyl silane coupling agent.

\subsection{Effect of reaction time on mercerization of Eulaliopsis binata fibers}

The effect of reaction time in the mercerization of Eulaliopsis binata fibers was studied in terms of percentage weight loss using the standard method(Singha et al., 2009) (Singha \& Thakur, 2009a) (Singha \& Thakur, 2009b). Figure 1 shows the effect of the mercerization (selected \% of $\mathrm{NaOH}$ was used in all the experiments). The maximum mercerization of Eulaliopsis binata fibers was found to occur at 270 minutes and after which it nearly saturates due to the trapping of 
sodium ions and other side reactions as these did not favor the further weight loss in the Eulaliopsis binata fibers. The mercerization of Eulaliopsis binata fibers facilitates the removal of impurities as well as definite amount of wax and lignin. After the optimization of time, mercerization of Eulaliopsis binata fibers was done using different concentration of $\mathrm{NaOH}$ starting from 3-15\%. The concentration of $\mathrm{NaOH}$ was varied in the range of 3-15\% because Eulaliopsis binata fibers are full of cellulose, hemicellulose and lignin along with various other constituents. These different components are strongly associated with each other and are difficult to separate/ remove using mild concentration of $\mathrm{NaOH}$. An appropriate amount of $\mathrm{NaOH}$ helps in the removal of undesired components from the cellulosic fibers and facilitates the modification through silane treatment/ graft copolymerization. In this work the concentration was carried using different $\%$ of $\mathrm{NaOH}$ and was optimized by studying the \% swelling behavior of Eulaliopsis binata fibers in different solvents; \% water uptake behavior of Eulaliopsis binata fibers and \% weight loss (in different chemicals) after treatment with fixed amount of silane as discussed in the following section.

\subsection{Silane treatment on mercerized Eulaliopsis binata fibers}

Vinyltrimethoxysilane treatment of different mercerized Eulaliopsis binata fibers was done as per the method reported in experimental section. Vinyltrimethoxysilane induced functionalization of Eulaliopsis binata fibers is a facile route to alter the surface characteristics of the raw fibers. However it is not as much effective as the graft copolymerization techniques where hydrophobic polymers are grafted onto the cellulosic fibers(Thakur, Singha, \& Thakur, 2014)(Thakur, Thakur, \& Singha, 2013). Functionalization of Eulaliopsis binata fibers with vinyltrimethoxysilane involves hydrolysis, condensation and bond formation stages, similar to 
the one reported for other natural cellulosic fibers with the difference in the structure of the silane coupling agent(Singha et al., 2009) (Singha \& Thakur, 2009a) (Singha \& Thakur, 2009b).

\subsection{Physico - chemical properties of Silane functionalized Eulaliopsis binata fibers}

Effect of vinyltrimethoxysilane treatment on the swelling behaviour, water uptake and chemical resistance of the pristine and functionalized Eulaliopsis binata fibers has been shown in the figure 2 to 5 respectively. It is obvious from the figure 2 that the raw Eulaliopsis binata fibers follows the trend in the swelling as $\mathrm{H}_{2} \mathrm{O}>\mathrm{CH}_{3} \mathrm{OH}>$ iso- $\mathrm{BuOH}$. This trend in the swelling behavior of raw fibers can be attributed the fact that raw cellulosic Eulaliopsis binata fibers contain abundant hydroxyl (-OH) groups and water molecules have greater affinity for these groups compared to the $\mathrm{CH}_{3} \mathrm{OH} \&$ iso- $\mathrm{BuOH}$. As a result of the greater affinity of water towards the hydroxyl groups of Eulaliopsis binata fibers, there is more penetration of water into these fibers rather than the other alcoholic solvents.

However after the silane functionalization, the Eulaliopsis binata fibers show less swelling due to the blockade of active functional groups cited by the vinyltrimethoxysilane(Singha et al., 2009) (Singha \& Thakur, 2009a) (Singha \& Thakur, 2009b). It is also evident from the figure that the $12 \%$ mercerized Eulaliopsis binata fibers followed by treatment with the vinyltrimethoxysilane show the lowest swelling behaviour. Similar behaviour was also observed in case of water uptake demonstrating that $12 \%$ mercerized fibers are most efficiently functionalized by vinyltrimethoxysilane coupling agents (figure 3). These functionalized Eulaliopsis binata fibers were also subjected to the study of chemical resistance behavior in the solutions of $1 \mathrm{~N} \mathrm{HCl}$ and $1 \mathrm{~N} \mathrm{NaOH}$. The resistance of the Eulaliopsis binata fibers towards chemicals was also found to be enhanced after silane functionalization (Figure 4- 
5). This behavior was also attributed to the blockage of active functional groups on the Eulaliopsis binata fibers vulnerable to the chemical attacks(Singha et al., 2009) (Singha \& Thakur, 2009a) (Singha \& Thakur, 2009b).

\subsection{Characterization of raw and grafted Eulaliopsis binata fibers}

The characterization of the Eulaliopsis binata fibers was carried out using FT-IR Spectroscopy, SEM and TGA analysis to ascertain the vinyltrimethoxysilane induced surface functionalization of these fibers. The FT-IR spectrum of the raw cellulosic Eulaliopsis binata fibers and vinyltrimethoxysilane functionalized fibers are shown in the figure 6(a-b). Figure 6 a shows the spectrum of raw Eulaliopsis binata fibers. The peaks at $3415 \mathrm{~cm}^{-1}, 1746 \mathrm{~cm}^{-1}$ and $1244 \mathrm{~cm}^{-1}$ in the spectrum of the Eulaliopsis binata fibers can be assigned to the bonded $\mathrm{OH}$ groups and the $\mathrm{C}-\mathrm{O}$ ring of lignin and carboxylic groups of pectin and hemicellulose(Thakur, Thakur, \& Gupta, 2013c)(Thakur, Thakur, \& Gupta, 2013e). Several other peaks in the spectrum of the Eulaliopsis binata fibers can be assigned to the basic constituents of any lignocellulosic fibers. However, after the functionalization of the Eulaliopsis binata fibers, some of the basic peaks of the fibers are disappeared while some due to silane groups are appeared. The peaks at $1746 \mathrm{~cm}^{-1}$ and $1244 \mathrm{~cm}^{-1}$ were found to be disappeared as a result of mercerization of these fibers. However, some new peaks as a result of the silane incorporation onto the Eulaliopsis binata fibers also appeared in the region from $750-1800 \mathrm{~cm}^{-1}$ further confirming the successful functionalization of these fibers through silane treatment(Singha et al., 2009) (Singha \& Thakur, 2009a) (Singha \& Thakur, 2009b). The peaks at $1575 \mathrm{~cm}^{-1}$ and $1490 \mathrm{~cm}^{-1}$ strongly demonstrated the presence of the silane functional groups on the Eulaliopsis binata fibers(Singha et al., 2009) (Singha \& Thakur, 2009a) (Singha \& Thakur, 2009b). 
Figure 7 (a-b) shows the SEM images of the raw and vinyltrimethoxysilane functionalized Eulaliopsis binata fibers. Both the images show the clear cut difference in the surface morphology of the fibers. It is observed from these image that the fibers surface changes significantly after the silane treatment and become almost rough. The changes in the morphology can be attributed to the removal of the impurities, wax, hemicellulose etc. from these fibers as well formation of silane coating the Eulaliopsis binata fibers surface.

Figure 8 (a-b) shows the thermograms of the raw and vinyltrimethoxysilane functionalized Eulaliopsis binata fibers surface. It is evident from the thermograms that the thermal degradation of Eulaliopsis binata fibers prior to and after silane functionalization covers a wide range of temperatures. For the raw Eulaliopsis binata fibers, the initial decomposition (IDT) temperature was found to be of $208{ }^{0} \mathrm{C}$ with a percent weight loss of $11 \%$. The final decomposition temperature (FDT) for these fibers was found to be $480{ }^{\circ} \mathrm{C}$ with a percent weight loss of $75 \%$. This thermal behaviour is similar to the other cellulosic fibers(Singha et al., 2009) (Singha \& Thakur, 2009a) (Singha \& Thakur, 2009b). After silane functionalization the modified fibers showed little variation in the thermal stability. The FDT of the vinyltrimethoxysilane functionalized Eulaliopsis binata fibers was found to be 491 0C. Thus both the SEM and TGA study further supports the FTIR data reconfirming the successful functionalization of the Eulaliopsis binata fibers through vinyltrimethoxysilane coupling agent.

\section{Conclusions}

Eulaliopsis binata fibers are one of the unexplored lignocellulosic materials having huge potential to be used in a number of applications. In this work, the surface functionalization of 
lignocellulosic Eulaliopsis binata fibers was carried out using mercerization followed by silane treatment using vinyltrimethoxysilane as a coupling agent. Subsequently, the effect of silane functionalization onto the physico-chemical behavior of the Eulaliopsis binata fibers was studied. It was observed from the study that the vinyltrimethoxysilane induced functionalization significantly affects the physico-chemical properties of the Eulaliopsis binata fibers. These surface modified fibers can be subsequently used as one of the component in the preparation of the green polymer composites.

\section{Acknowledgement}

Authors wish to thanks their parental institutes for providing the necessary facilities to accomplish the present research work.

\section{REFERENCES}

Basta, A. H., El-Saied, H., El-Hadi, O., \& El-Dewiny, C. (2013). Evaluation of Rice Straw-Based Hydrogels for Purification of Wastewater. Polymer-Plastics Technology and Engineering, 52(11), 1074-1080. doi:10.1080/03602559.2013.806548

Bharti, S., Mishra, S., \& Sen, G. (2013). Ceric ion initiated synthesis of polyacrylamide grafted oatmeal: Its application as flocculant for wastewater treatment. Carbohydrate Polymers, 93(2), 528-536. doi:10.1016/j.carbpol.2012.11.072 
Chen, C., \& Gao, Y. (2007a). Electrochemical characteristics of polyaniline electrosynthesized in the presence of neutral red. Materials Chemistry and Physics, 102(1), 24-30. doi:10.1016/j.matchemphys.2006.10.005

Chen, C., \& Gao, Y. (2007b). Electrochemical polymerization of neutral red in the presence of sulfoferrocenecarboxylic acid. Polymer, 48(19), 5572-5580. doi:10.1016/j.polymer.2007.06.072

Dhakal, H. N., Zhang, Z. Y., \& Bennett, N. (2012). Influence of fibre treatment and glass fibre hybridisation on thermal degradation and surface energy characteristics of hemp/unsaturated polyester composites. Composites Part B-Engineering, 43(7), 27572761. doi:10.1016/j.compositesb.2012.04.036

Dhakal, H. N., Zhang, Z. Y., Guthrie, R., MacMullen, J., \& Bennett, N. (2013). Development of flax/carbon fibre hybrid composites for enhanced properties. Carbohydrate Polymers, 96(1), 1-8. doi:10.1016/j.carbpol.2013.03.074

Dhakal, H. N., Zhang, Z. Y., Reis, P. N. B., Surip, S. N., \& Bennett, N. (2012). Evaluation of Water Absorption Damage in Hemp Fibre Reinforced Unsaturated Polyester Composites by the Nanoindentation Testing. Journal of Biobased Materials and Bioenergy, 6(4), 493-499. doi:10.1166/jbmb.2012.1230

Ding, G., Cho, C. M., Chen, C., Zhou, D., Wang, X., Tan, A. Y. X., Lu, X. (2013). Black-totransmissive electrochromism of azulene-based donor-acceptor copolymers complemented by poly (4-styrene sulfonic acid)-doped poly (3, 4ethylenedioxythiophene). Organic Electronics, 14(11), 2748-2755. doi:10.1016/j.orgel.2013.07.037 
Jawaid, M., \& Khalil, H. P. S. A. (2011). Cellulosic/synthetic fibre reinforced polymer hybrid composites: A review. Carbohydrate Polymers, 86(1), 1-18. doi:10.1016/j.carbpol.2011.04.043

Khalil, H. P. S. A., Jawaid, M., Firoozian, P., Amjad, M., Zainudin, E., \& Paridah, M. T. (2013). Tensile, Electrical Conductivity, and Morphological Properties of Carbon Black-Filled Epoxy Composites. International Journal of Polymer Analysis and Characterization, 18(5), 329-338. doi:10.1080/1023666X.2013.782841

Khoathane, M. C., Vorster, O. C., \& Sadiku, E. R. (2008). Hemp fiber-reinforced 1pentene/polypropylene copolymer: The effect of fiber loading on the mechanical and thermal characteristics of the composites. Journal of Reinforced Plastics and Composites, 27(14), 1533-1544. doi:10.1177/0731684407086325

Lin, M.-F., \& Lee, P. S. (2013). Formation of PVDF-g-HEMA/BaTiO3 nanocomposites via in situ nanoparticle synthesis for high performance capacitor applications. Journal of Materials Chemistry A, 1(46), 14455-14459. doi:10.1039/C3TA13190D

Lin, M.-F., Thakur, V. K., Tan, E. J., \& Lee, P. S. (2011a). Dopant induced hollow BaTiO3 nanostructures for application in high performance capacitors. Journal of Materials Chemistry, 21(41), 16500. doi:10.1039/c1jm12429c

Lin, M.-F., Thakur, V. K., Tan, E. J., \& Lee, P. S. (2011b). Surface functionalization of BaTiO3 nanoparticles and improved electrical properties of $\mathrm{BaTiO} 3 /$ polyvinylidene fluoride composite. RSC Advances, 1(4), 576. doi:10.1039/c1ra00210d

Maheswari, C. U., Reddy, K. O., Muzenda, E., Shukla, M., \& Rajulu, A. V. (2013). Mechanical Properties and Chemical Resistance of Short Tamarind Fiber/Unsaturated Polyester Composites: Influence of Fiber Modification and Fiber Content. International Journal of 
Polymer Analysis and Characterization, 18(7), 520-533. doi:10.1080/1023666X.2013.816073

Mishra, S., Rani, G. U., \& Sen, G. (2012). Microwave initiated synthesis and application of polyacrylic acid grafted carboxymethyl cellulose. Carbohydrate Polymers, 87(3), 22552262. doi:10.1016/j.carbpol.2011.10.057

Pilla, S., Gong, S., O’Neill, E., Rowell, R. M., \& Krzysik, A. M. (2008). Polylactide-pine wood flour composites. Polymer Engineering and Science, 48(3), 578-587. doi:10.1002/pen.20971

Pilla, S., Gong, S., O’Neill, E., Yang, L., \& Rowell, R. M. (2009). Polylactide-Recycled Wood Fiber Composites. Journal of Applied Polymer Science, 111(1), 37-47. doi:10.1002/app.28860

Pinto, M. A., Chalivendra, V. B., Kim, Y. K., \& Lewis, A. F. (2013). Effect of surface treatment and Z-axis reinforcement on the interlaminar fracture of jute/epoxy laminated composites. Engineering Fracture Mechanics, 114, 104-114. doi:10.1016/j.engfracmech.2013.10.015

Pinto, M. A., Chalivendra, V. B., Kim, Y. K., \& Lewis, A. F. (2014). Evaluation of surface treatment and fabrication methods for jute fiber/epoxy laminar composites. Polymer Composites, 35(2), 310-317. doi:10.1002/pc.22663

Rani, G. U., Mishra, S., Sen, G., \& Jha, U. (2012). Polyacrylamide grafted Agar: Synthesis and applications of conventional and microwave assisted technique. Carbohydrate Polymers, 90(2), 784-791. doi:10.1016/j.carbpol.2012.05.069

Rani, P., Sen, G., Mishra, S., \& Jha, U. (2012). Microwave assisted synthesis of polyacrylamide grafted gum ghatti and its application as flocculant. Carbohydrate Polymers, 89(1), 275281. doi:10.1016/j.carbpol.2012.03.009 
Sabo, R., Basta, A. H., \& Winandy, J. E. (2013). Integrated Study of the Potential Application of Remediated CCA Treated Spruce Wood in MDF Production. Industrial \& Engineering Chemistry Research, 52(26), 8962-8968. doi:10.1021/ie400536d

Singha, A. S., \& Thakur, V. K. (2009a). Morphological, Thermal, and Physicochemical Characterization of Surface Modified Pinus Fibers. International Journal of Polymer Analysis and Characterization, 14(3), 271-289. doi:10.1080/10236660802666160

Singha, A. S., \& Thakur, V. K. (2009b). Synthesis and Characterizations of Silane Treated Grewia optiva Fibers. International Journal of Polymer Analysis and Characterization, 14(4), 301-321. doi:10.1080/10236660902871470

Singha, A. S., Thakur, V. K., Mehta, I. K., Shama, A., Khanna, A. J., Rana, R. K., \& Rana, A. K. (2009). Surface-Modified Hibiscus sabdariffa Fibers: Physicochemical, Thermal, and Morphological Properties Evaluation. International Journal of Polymer Analysis and Characterization, 14(8), 695-711. doi:10.1080/10236660903325518

Thakur, V. K., Singha, A. S., Kaur, I., Nagarajarao, R. P., \& Liping, Y. (2010). Silane Functionalization of Saccaharum cilliare Fibers: Thermal, Morphological, and Physicochemical Study. International Journal of Polymer Analysis and Characterization, 15(7), 397-414. doi:10.1080/1023666X.2010.510106

Thakur, V. K., Singha, A. S., \& Thakur, M. K. (2011). Biopolymers Based Green Composites: Mechanical, Thermal and Physico-chemical Characterization. Journal of Polymers and the Environment, 20(2), 412-421. doi:10.1007/s10924-011-0389-y

Thakur, V. K., Singha, A. S., \& Thakur, M. K. (2012). Green Composites from Natural Fibers: Mechanical and Chemical Aging Properties. International Journal of Polymer Analysis and Characterization, 17(6), 401-407. doi:10.1080/1023666X.2012.668665 
Thakur, V. K., Singha, A. S., \& Thakur, M. K. (2013a). Fabrication and Physico-Chemical Properties of High-Performance Pine Needles/Green Polymer Composites. International Journal of Polymeric Materials, 62(4), 226-230. doi:10.1080/00914037.2011.641694

Thakur, V. K., Singha, A. S., \& Thakur, M. K. (2013b). Natural Cellulosic Polymers as Potential Reinforcement in Composites: Physicochemical and Mechanical Studies. Advances in Polymer Technology, 32(S1), E427-E435. doi:10.1002/adv.21290

Thakur, V. K., Singha, A. S., \& Thakur, M. K. (2014). Pressure Induced Synthesis of EA Grafted Saccaharum cilliare Fibers. International Journal of Polymeric Materials and Polymeric Biomaterials, 63(1), 17-22. doi:10.1080/00914037.2013.769243

Thakur, V. K., Thakur, M. K., \& Gupta, R. K. (2013a). Development of functionalized cellulosic biopolymers by graft copolymerization. International Journal of Biological Macromolecules, 62, 44-51. doi:10.1016/j.ijbiomac.2013.08.026

Thakur, V. K., Thakur, M. K., \& Gupta, R. K. (2013b). Graft copolymers from cellulose: Synthesis, characterization and evaluation. Carbohydrate Polymers, 97(1), 18-25. doi:10.1016/j.carbpol.2013.04.069

Thakur, V. K., Thakur, M. K., \& Gupta, R. K. (2013c). Graft Copolymers from Natural Polymers Using Free Radical Polymerization. International Journal of Polymer Analysis and Characterization, 18(7), 495-503. doi:10.1080/1023666X.2013.814241

Thakur, V. K., Thakur, M. K., \& Gupta, R. K. (2013d). Rapid synthesis of graft copolymers from natural cellulose fibers. Carbohydrate Polymers, 98(1), 820-828. doi:10.1016/j.carbpol.2013.06.072

Thakur, V. K., Thakur, M. K., \& Gupta, R. K. (2013e). Synthesis of lignocellulosic polymer with improved chemical resistance through free radical polymerization. International Journal of Biological Macromolecules, 61, 121-126. doi:10.1016/j.ijbiomac.2013.06.045 
Thakur, V.K., Thakur, M.K., \& Gupta, R.K. (2013f). Eulaliopsis binata: Utilization of Waste Biomass in Green Composites. In Green Composites from Natural Resources (pp. 125132). CRC Press. Retrieved from http://www.crenetbase.com/doi/abs/10.1201/b16076-7

Thakur, V. K., Thakur, M. K., \& Gupta, R. K. (2014). Graft copolymers of natural fibers for green composites. Carbohydrate Polymers, $\quad$ 104, 87-93. doi:10.1016/j.carbpol.2014.01.016

Thakur, V. K., Thakur, M. K., \& Singha, A. S. (2013). Free Radical-Induced Graft Copolymerization onto Natural Fibers. International Journal of Polymer Analysis and Characterization, 18(6), 430-438. doi:10.1080/1023666X.2013.814026

Zhou, D., Zhou, R., Chen, C., Yee, W.-A., Kong, J., Ding, G., \& Lu, X. (2013). Non-Volatile Polymer Electrolyte Based on Poly (propylene carbonate), Ionic Liquid, and Lithium Perchlorate for Electrochromic Devices. Journal of Physical Chemistry B, 117(25), 77837789. doi: $10.1021 / \mathrm{jp} 4021678$ 


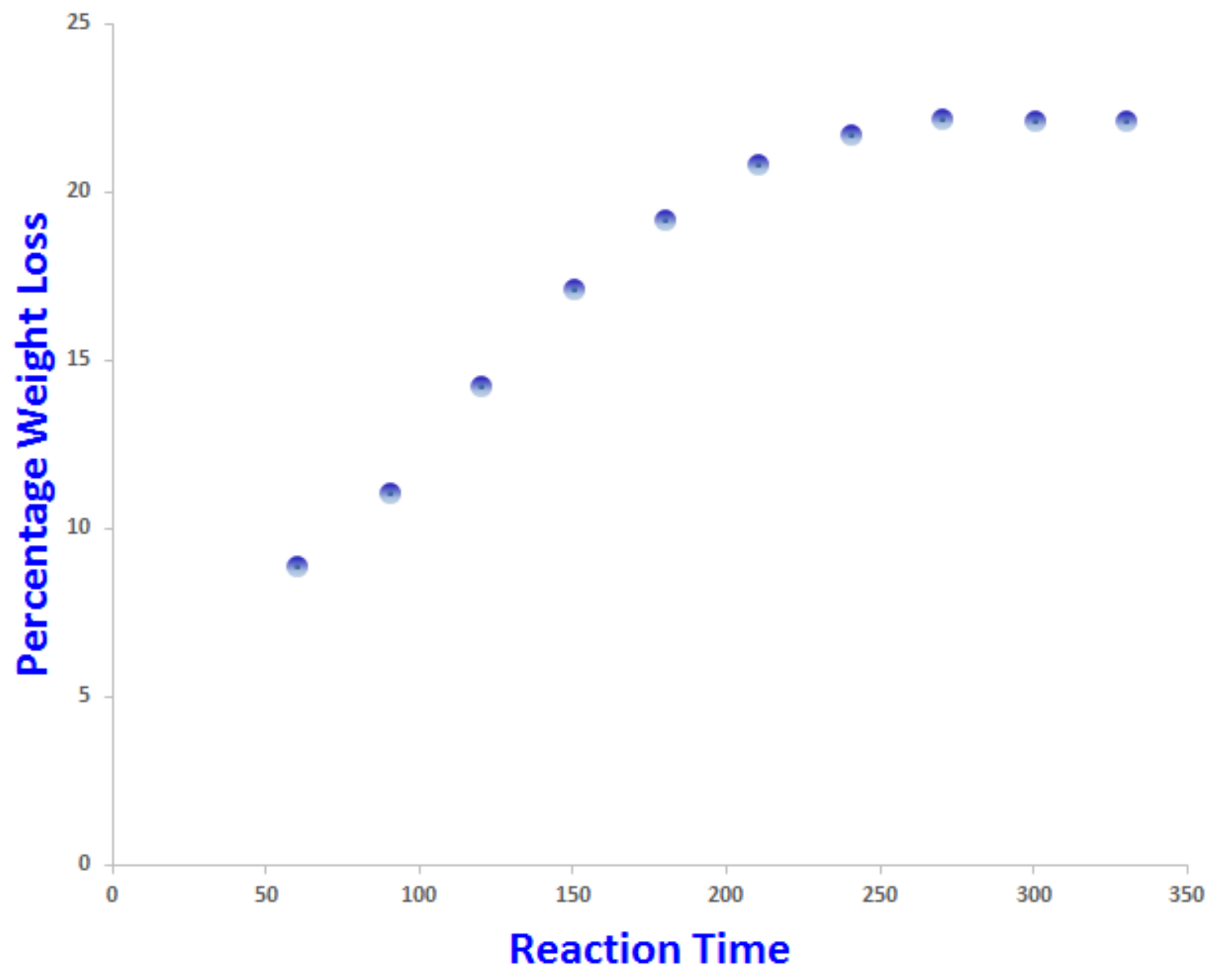

Figure 1- Effect of reaction time on mercerization of Cellulosic Eulaliopsis binata fibers 


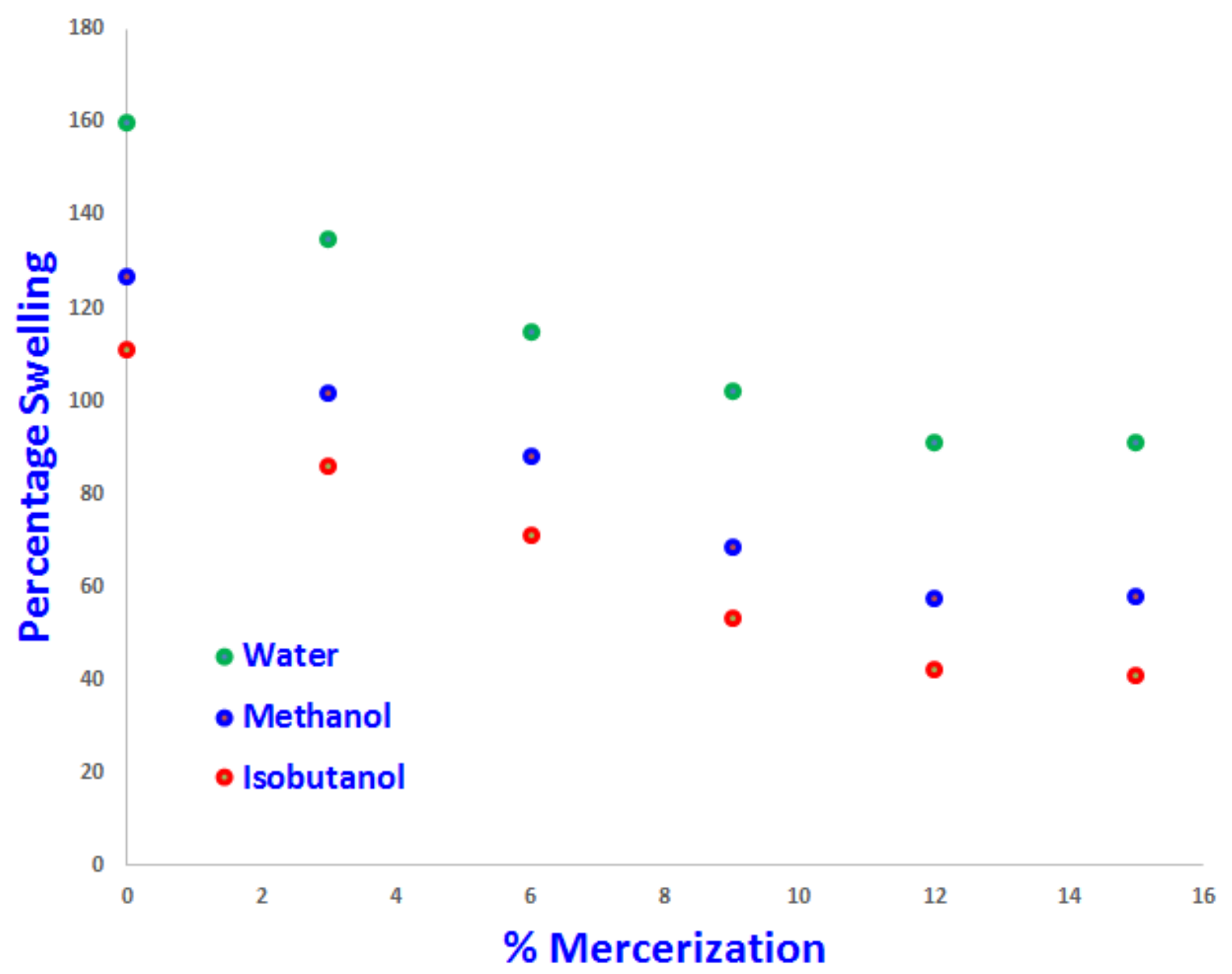

Figure 2 Effect of silane treatment on \% swelling behavior of Eulaliopsis binata fibers in different solvents 


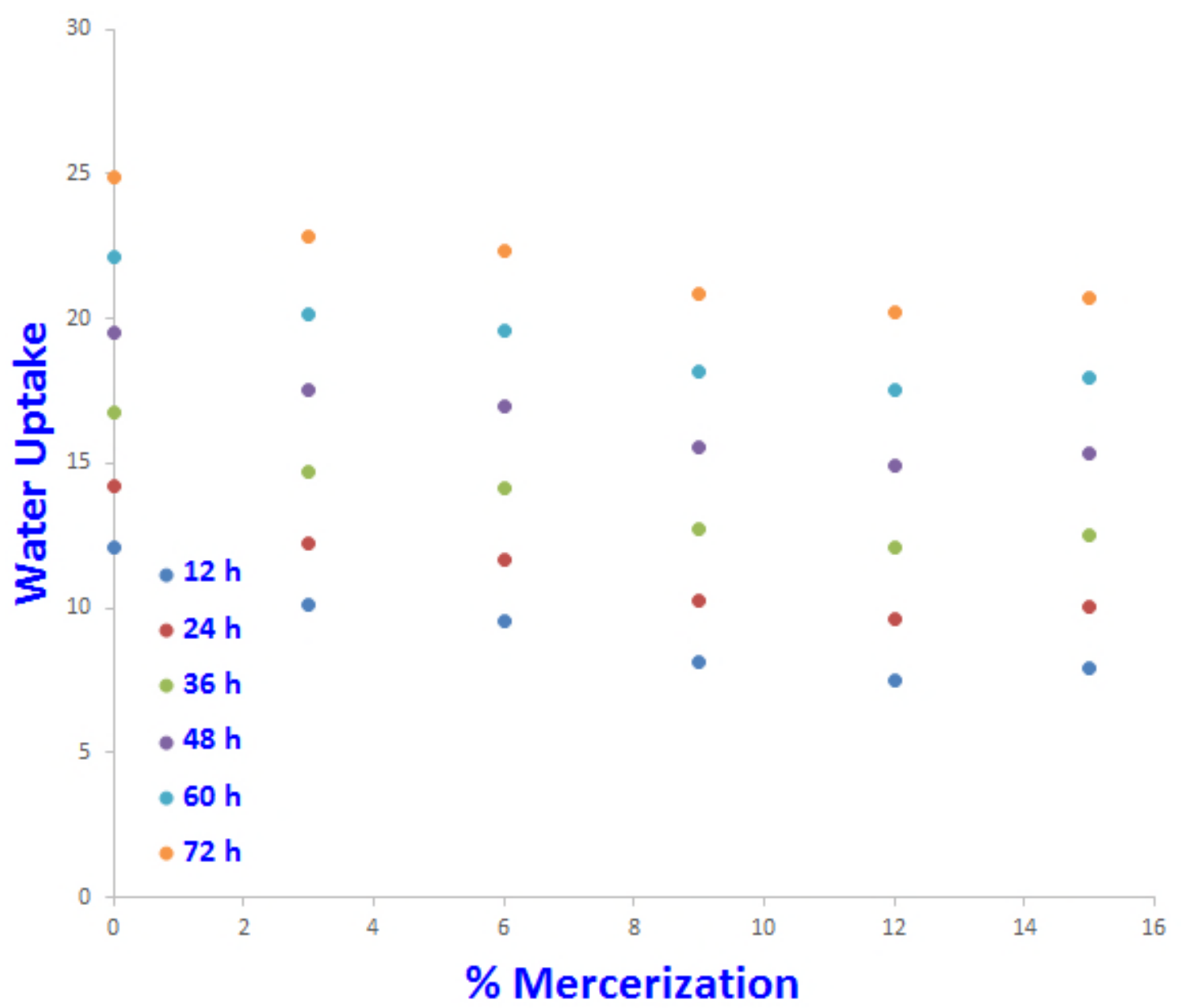

Figure 3 Effect of silane treatment on \% water uptake behavior of Eulaliopsis binata fibers. 


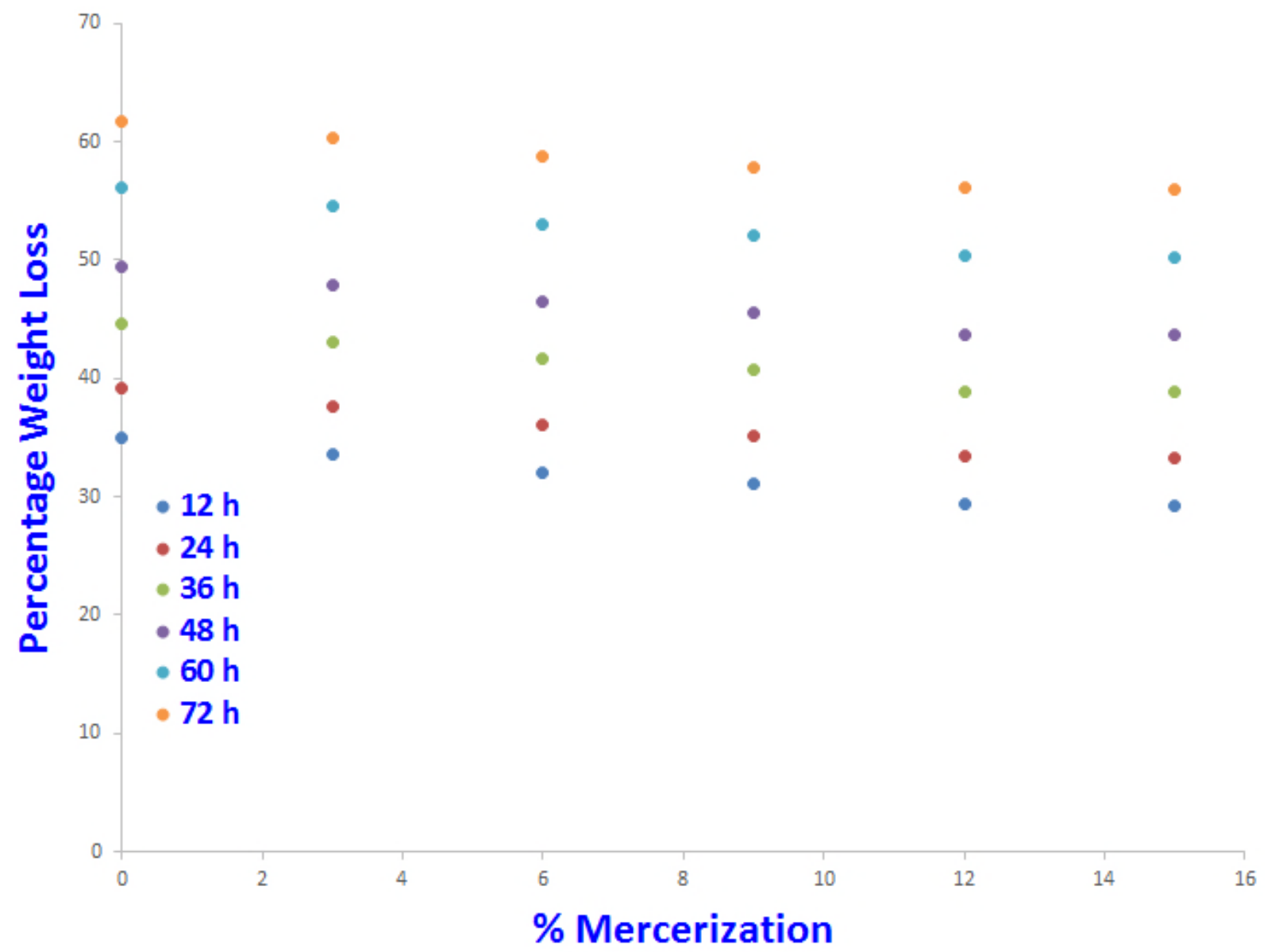

Figure 4 Effect of silane treatment on acid resistance behavior of raw and grafted Cellulosic Eulaliopsis binata fibers in $1 \mathrm{~N} \mathrm{HCl}$ at different time intervals 


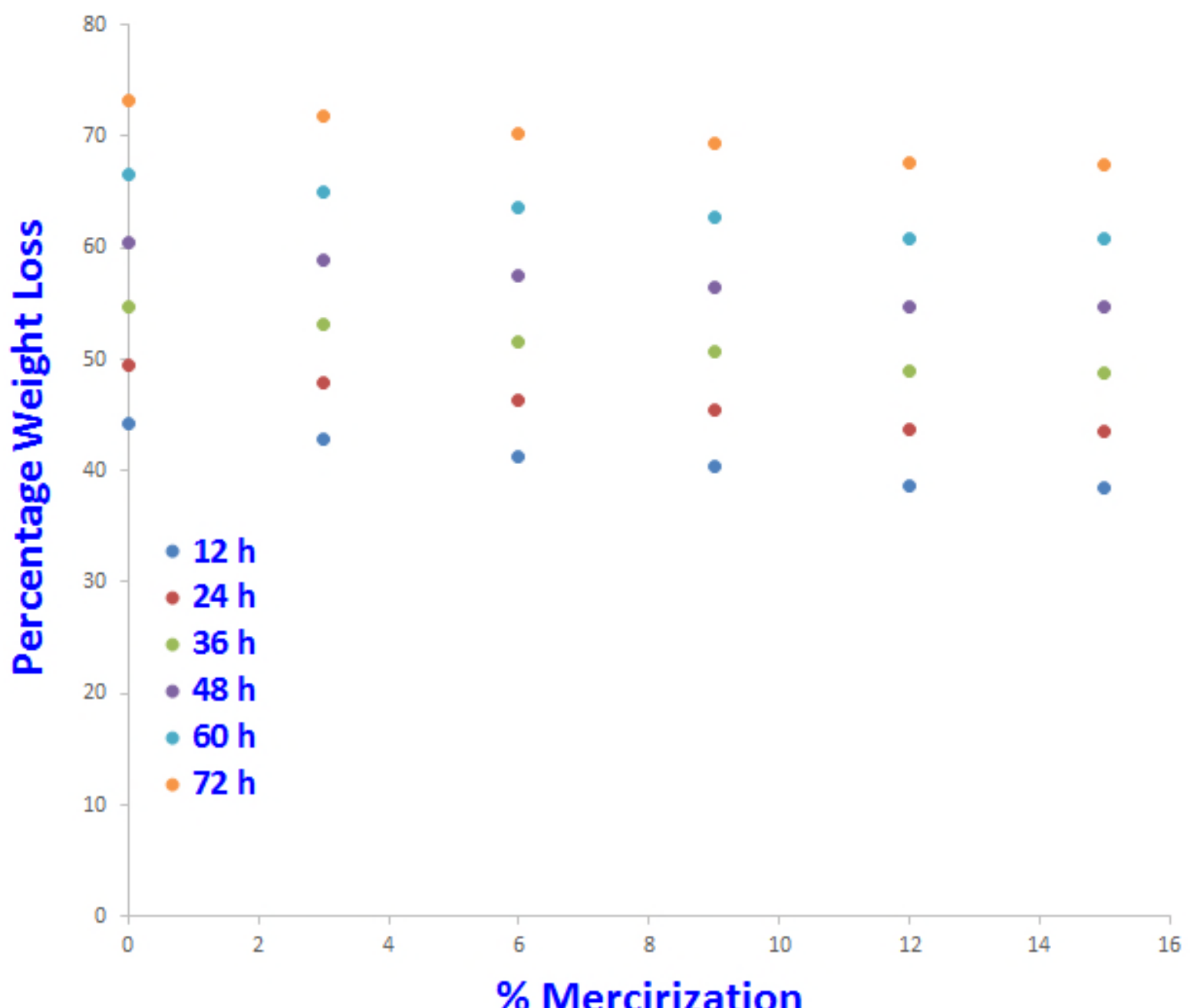

Figure 5 Effect of silane treatment on base resistance behavior of raw and grafted Cellulosic Eulaliopsis binata fibers in $1 \mathrm{~N} \mathrm{NaOH}$ at different time intervals. 


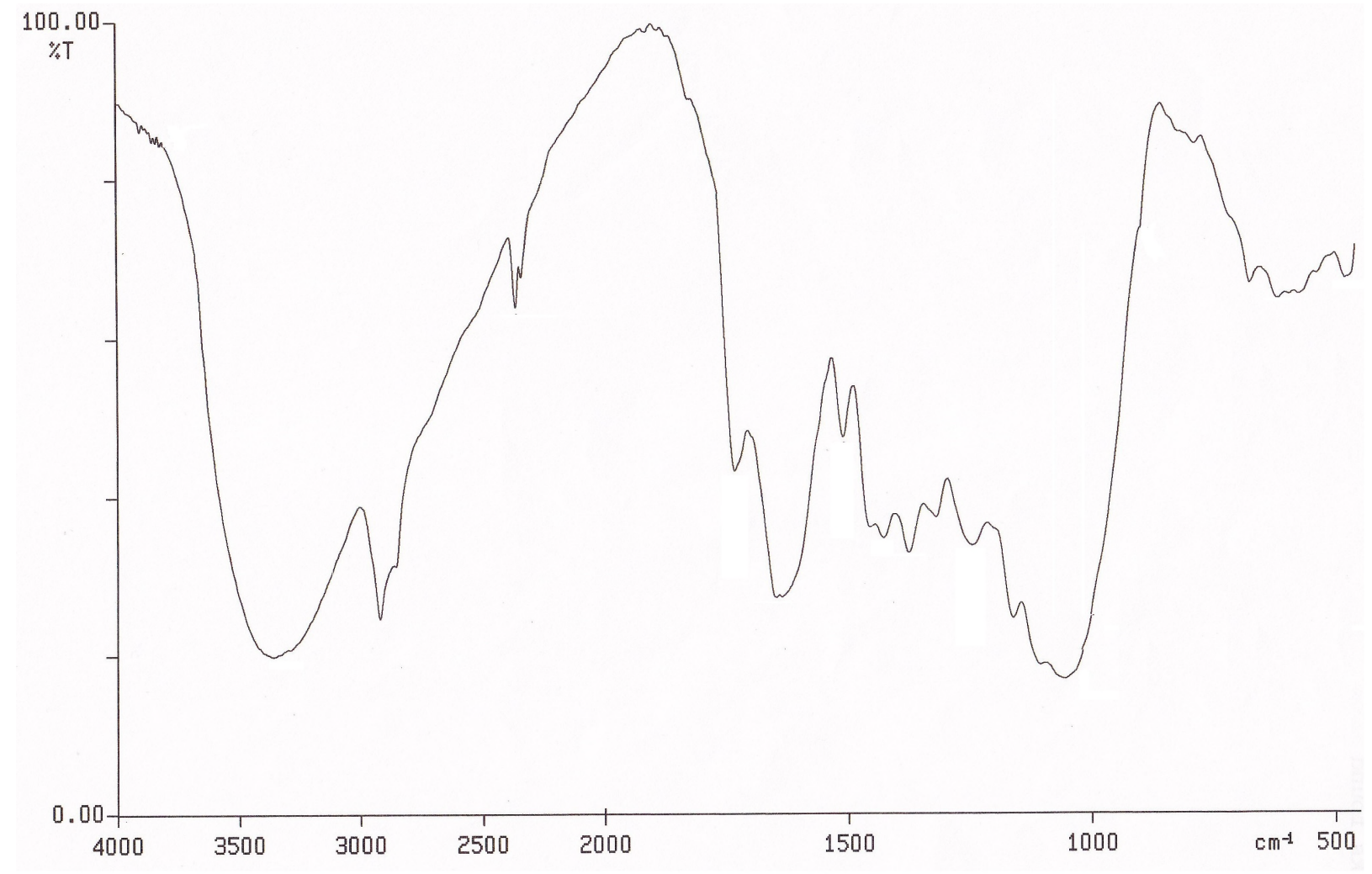

Figure 6(a) FTIR spectrum of raw cellulosic Eulaliopsis binata fibers. 


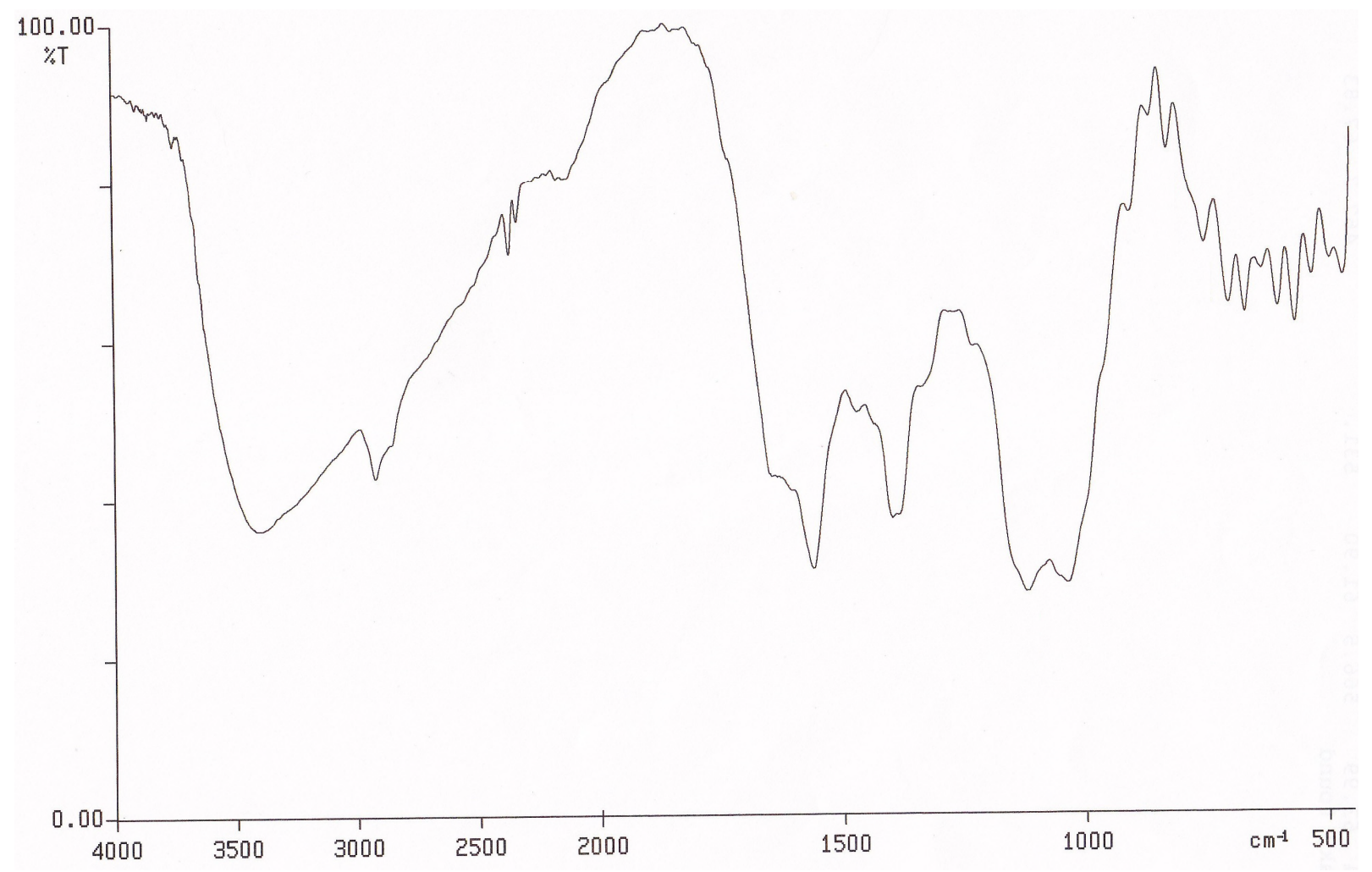

Figure 6 (b) FTIR spectrum of silane treated cellulosic Eulaliopsis binata fibers 


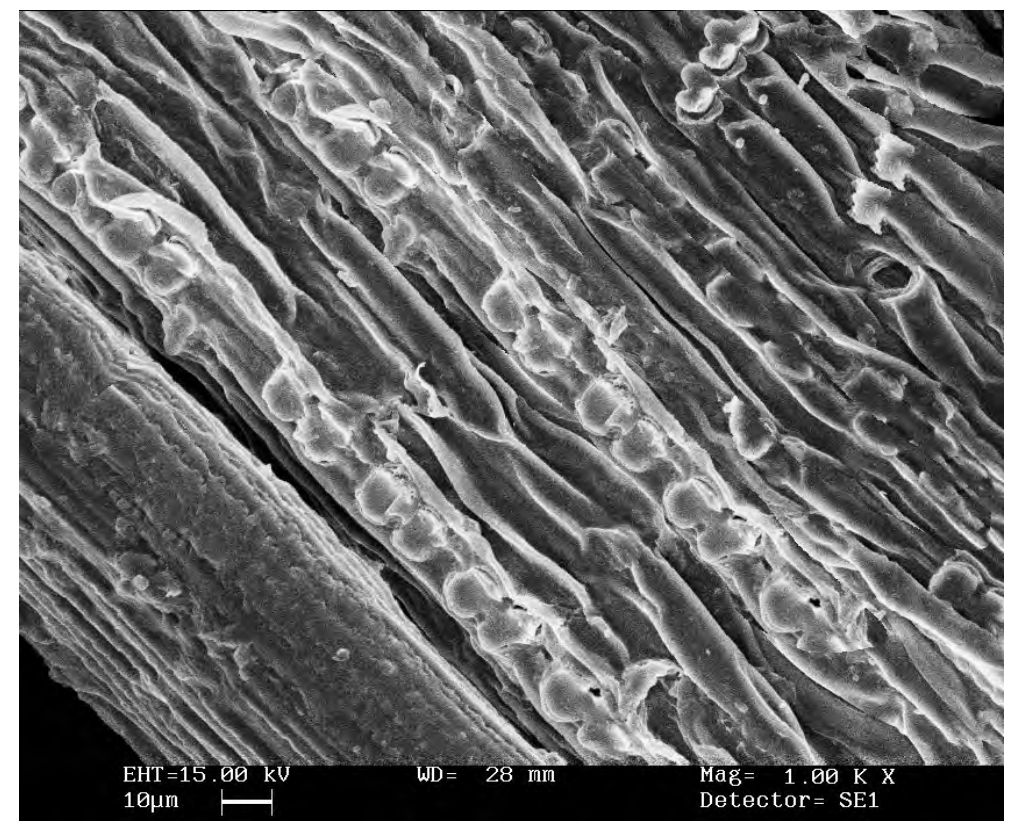

(a)

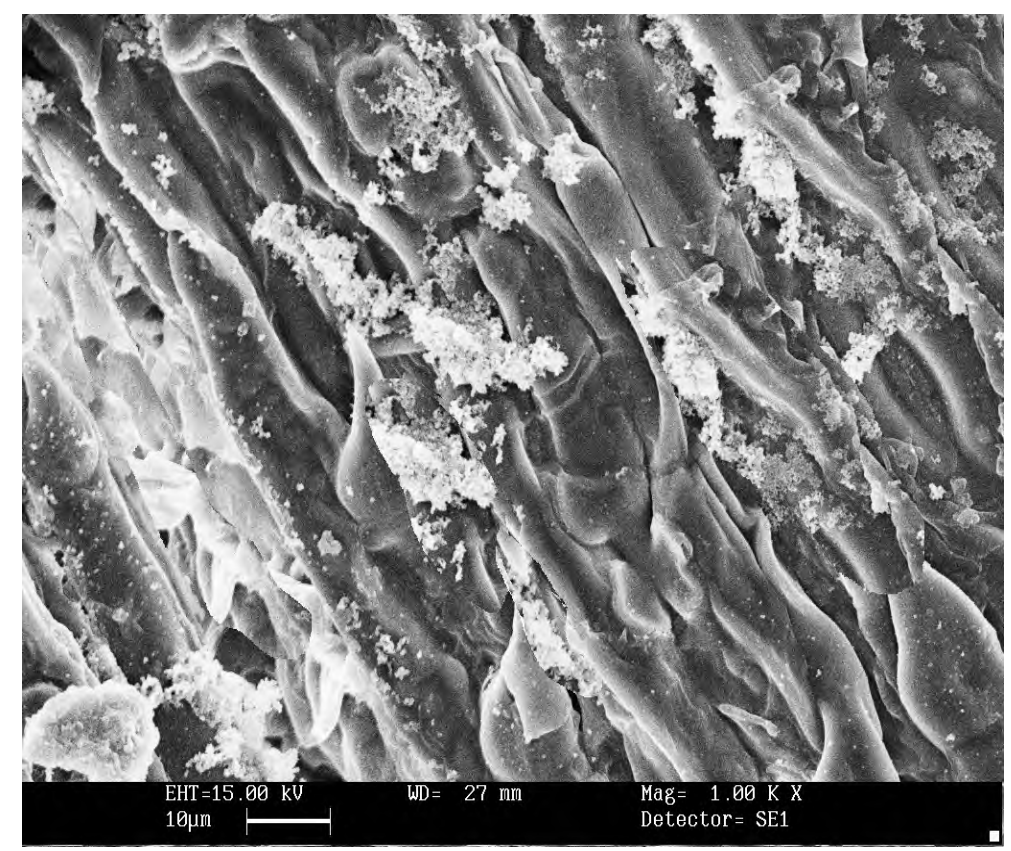

(b)

Figure 7- Scanning electron micrographs of (a) raw cellulosic Eulaliopsis binata fibers (b) silane treated cellulosic Eulaliopsis binata fibers. 


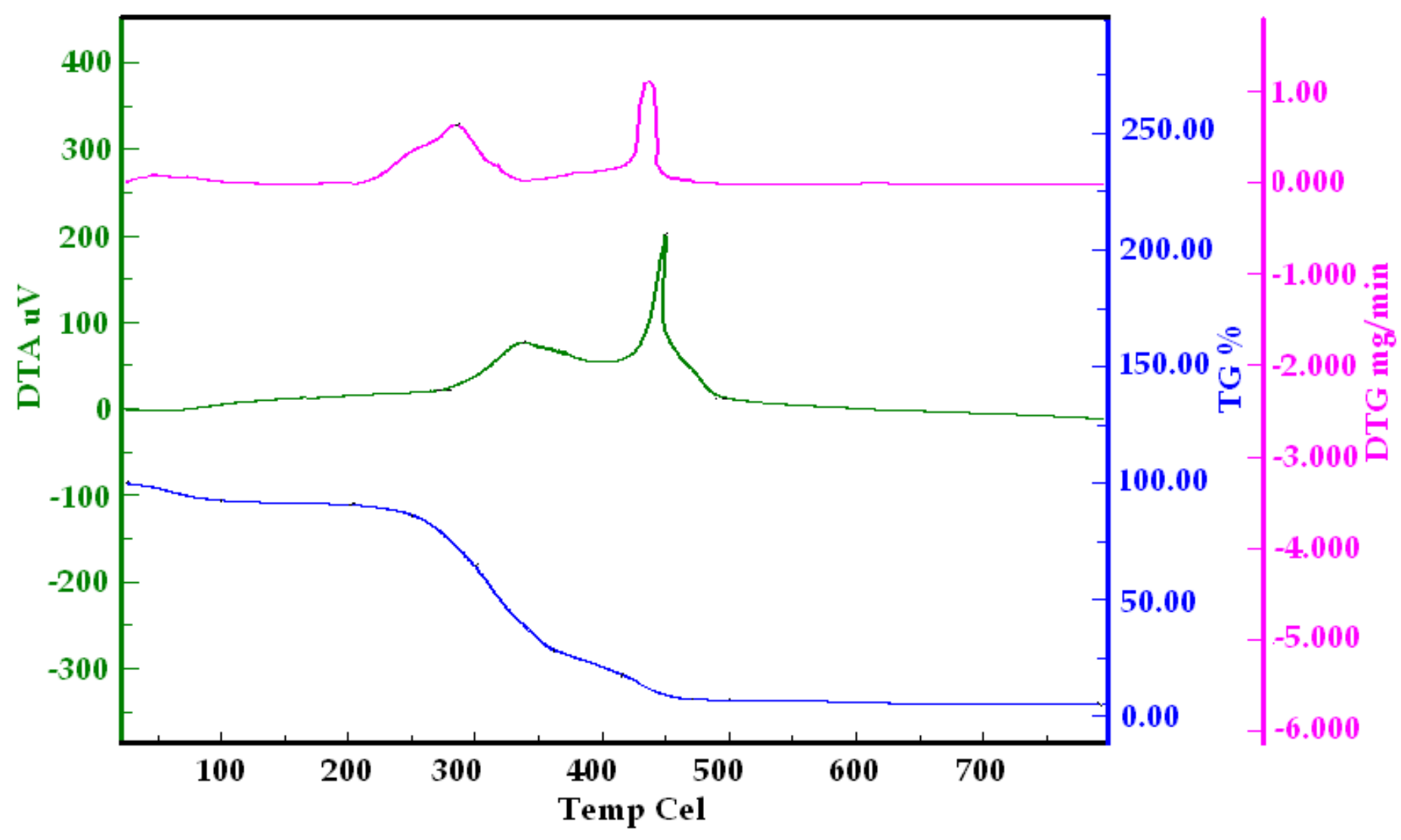

Figure 8 (a) - Thermogravimetric analysis of raw cellulosic Eulaliopsis binata fibers 


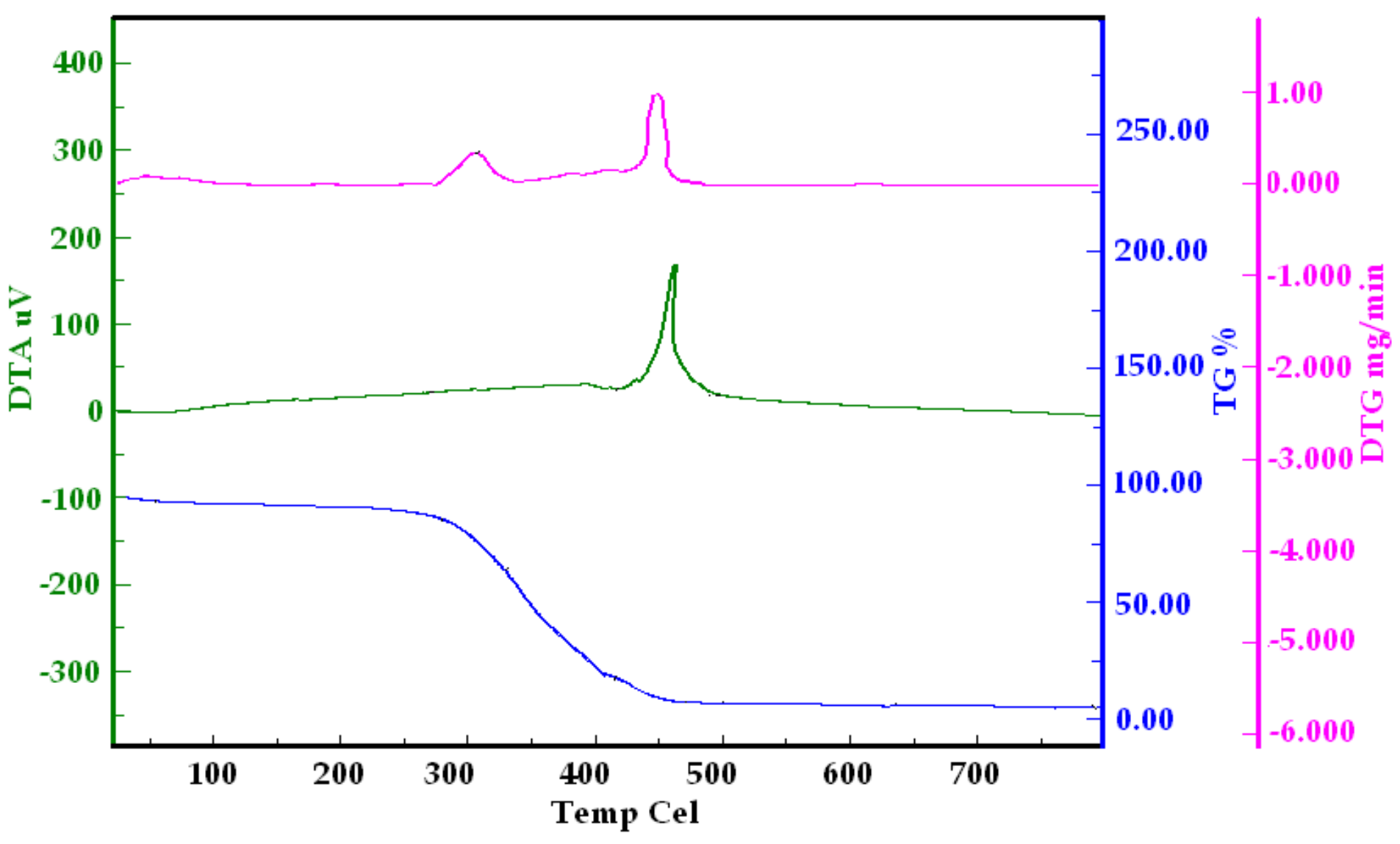

Figure 8 (b) - Thermogravimetric analysis of silane treated cellulosic Eulaliopsis binata fibers 
2014-05-26

\title{
Surface modification of cellulose using silane coupling agent
}

\author{
Thakur, Manju Kumari
}

Elsevier

Thakur MK, Gupta RK, Thakur VK. (2014) Surface modification of cellulose using silane coupling agent. Carbohydrate Polymers, Volume 111, October 2014, pp. 849-855

https://doi.org/10.1016/j.carbpol.2014.05.041

Downloaded from Cranfield Library Services E-Repository 